which should be elucidated as a preliminary to further experimentation.

The research which we have conducted has shown that the actual phenomena of mitosis in human lymphocytes can now be demonstrated. The remarkable differences between these cells and the other leucocytes are now apparent. Lymphocytes respond to the auxetic and endeavour to reproduce themselves, whereas leucocytes do not. The cytoplasmic (A'ltmann's) granules of leucocytes can be stained without harm to the lives of the cells. The nuclear granules of the lymphocytes, on the other hand, are composed of chromatin, and somatic death occurs when they stain. The fact that the nucleoli of lymphocytes form the centrosomes is also interesting, but not unique, from a cytological point of view.

In concluding the present report, we may mention that extracts from organs other than haemal glands are effective in assisting to promote mitotic changes-for instance, suprarenal gland and the udder of a ewe-but thyroid gland and udders of lambs do not appear to contain the necessary accelerator of reproduction, and, furthermore, they do not prevent it. The mitoses seen when chromatin extract is used sometimes show a remarkable "lop-sided" appearance, but we have been unable to satisfy ourselves that this was connected with sinapsis, although suggestive of it. In performing the experiments to produce the mitoses which we have described care and accuracy in carrying out details are essential, for unless the alkalinity of the jelly is correct and the temperature accurate no mitoses will be seen, and the cells will remain at rest. The mitoses occur only at a temperature of $37^{\circ} \mathrm{C}$. and in the few moments which immediately precede death and the staining of the chromosomes.

REFERENCES.
1 H. Ross, Journal of Physioloyy, vol. xxxvii, No. $4 . \quad 2$ H. Ross, Lancet January 23rd, 1909. 3 July 27th, 1907. $\mathbf{H}$ H. Ross, ibid.. Jass, Moore and Walker, ibid., Roy. Soc. Proc., B. 81, No. 546, 1909 . 6 H. Ross, ibid., B. 81, No. 549, 1909. 7 Ross and Macalister, BRITISH MEDICAL JouRNAL, January 16th, 1909. 8 Macalister and Ross, Proc. Roy. Soc. Med., December, 1908. $9 \mathrm{H}$. Ross, the Lancet, January 30th. 1909. 10 Bashford, ibid., Septem ber 4th, 1909. $11 \mathrm{H}$. Rnss, ibid., February 6th, 1909. 12 Bashford and Murray, Roy. Soc. Proc., B. 81, 1909. 13 Farmer, Moore and Walker First Report of Cytological Investigation on Cancer, Liverpool, 1906.

\section{THE DIAGNOSIS OF MALIGNANT DISEASE} BY MEANS OF THE ANTITRYPTIC INDEX.

\author{
BY
}

HUGH WANSEY BAYLY, M.A.CAMB., M.R C.S., L.R.C.P.

PATHOLOGIST TO THE LONDON LOCK HOSPITALS, ASSISTANT IN THE BACTERIOLOGICAT DEPARTMENT OF ST, GEORGE'S HOSPITAI.

(From the Bacteriological Laboratories, St. George's Hospital.)

By the antitryptic index is meant the power of inhibiting tryptic digestion possessed by any given serum, compared with such inlibitory power possessed by a normal standard serum; or, in other words, the ratio of the powers possessed by different serums for neutralizing tryptic ferments.

Dr. F. L. Golla, ${ }^{1}$ in a paper on the clinical value of the antitryptic index in tuberculosis, pointed out that the variations in the antitryptic powers of serums were not only interesting laboratory phenomena but could be applied for clinical and diagnostic purposes. He employed the electrical conductivity method introduced by Victor Henri for the study of ferment action, and it is this method that I have used throughout, employing the technique described by Dr. Golla in his paper.

Mr. E. C. Hort in the same month published a paper ${ }^{2}$ on a chemical method for estimating the antitryptic index, especially in relation to diagnosis of malignant disease In July, 1909, Mr. Hort read a paper before the British Medical Association at Belfast on the diagnosis of cancer by examination of the blood. Here also he used the chemical method, and found that the antitryptic index was raised in 94 per cent. of cases of malignant disease.

Dr. Golla, Mr. Hort, and myself, working independently, have observed that most processes involving cell destruction, whether bacterial, mechanical, or cytolytic, produce a raising of the antitryptic index. The phenomenon can, therefore, be in no way regarded as specific, but this lack of specificity, though it narrows the scope of the reaction, still leaves an ample margin where its utility as an aid to diagnosis in malignant disease is clearly shown.

Thus, in the absence of bacterial infection symptoms of obstruction or pressure accompanied by a raised antitryptic index would indicate the presence of a malignant rather than a benign growth or aneurysm, and a normal index would contraindicate the presence of malignant disease.

It has been observed that the serum of cases of gastric ulcer give a nearly normal reaction, and a diagnosis between this condition and carcinoma of the stomach can therefore be facilitated by the antitryptic test.

Perhaps it may be useful to give a fow more details as to quantities than were mentioned in Dr. Golla's paper. I have used $5 \mathrm{c.cm}$. of the formalized casein solution, $0.5 \mathrm{c.um}$. of serum, and $2.5 \mathrm{c.cm}$. of a fresh and filtered, roughly 10 per cent. solution of pure active pancreatin. The observations were made after the mixture had been in the thermostat ten minutes and four and a half hours, which I found to give the maximum of variation. All results were corrected for the different electrical capacities of the bottles, and the majority of the estimations were deter. mined in duplicate. Instead of expressing the index as Dr. Golla has by + and - signs, I have used the same notation as that employed for the opsonic index and taken 1 to express normal, so that an index expressed by $\mathrm{Dr}$. Golla's +0.6 is in my notation 1.6 and one of -0.6 is 0.4

My results confirm those obtained by Mr. Hort with the chemical method, but as the chemical method depends on the matching of shades of colour, I think that the electrical method, which registers quite clearly and un. mistakably very minute differences in the amount of digestion, will be found to be both the more reliable and easier for those without very much practical chemical experience.

Dr. Golla has lately introduced a new method by which he estimates the antitryptic index by means of the viscosity of the serum; but as the estimation of rate of flow requires accurate measurement of quantity, space, and time, there is ample opportunity for errors in observa. tion in unskilled hands. With the electrical method, however, providing that the bottles are standardized sufficiently often, there seems to be but little opportunity for experimental error.
Site of Cancer.

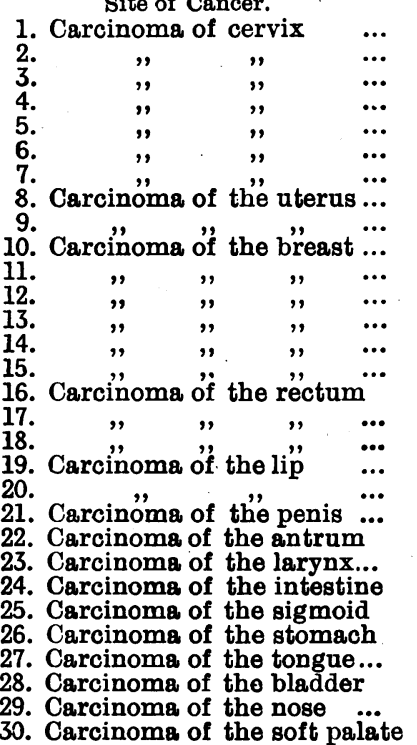

Antitryptic Index.

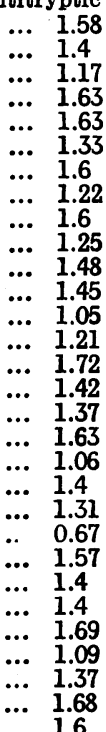

of the 30 cases of cancer that I have given above 26 (86 per cent.) show a markedly raised index. Of the remaining 4 cases, $3(13,19,27),=10$ per cent., show practically normal indices, and one (22) shows a sub. normal index. This case was a very advanced one, and died before I had the opportunity of obtaining another specimen. The section showed the tumour to be an epithelioma. Of the 3 cases showing normal indices, No. 13 was an early case ten days after operation; No. 19 was an early case before operation; and No. 27 was awaiting removal of the tongue. 
If bacterial infection is present, this method at the moment cannot claim any great diagnostic value', but in the absence of such infection its great help in affording a diagnosis between malignant disease and gastric or intestinal ulcers, non-malignant strictures, áneurysms, nonmalignant conditions of the bladder (such as stone), or non-malignant pelvic conditions in the female, is apparent. The negative evidence afforded by a normal antitryptic index is also of very great value in excluding malignant disease.

My thanks are due to Dr. Lunn and Dr. Roper, of the St. Marylebone Infirmary; Dr. Potter, of the Kensington Infirmary; and Dr. Coulson, of the St. George's (Hanover Square)-Infirmary, for their kindness in giving me access to their cases.

ReFerences.

1 Lancet, April 3rd, 1909. 2 Clinical Journal, April, 1909.

\section{AN INVESTIGATION OF SERRATUS MAGNUS INFECTION IN CANCER OF THE} BREAST.

By T. BONHÔTE HENDERSON, M.A., M.B., B.Ch.Oxon., F.R.C.S.Eng.,

SURGICAL REGISTRAR TO THE CANCER HOSPITAL, BROMPTON.

IT has been my misfortune during a period of more than two and a half years to record the notes of 75 cases of recurrent mammary cancers. The unhappy victims have come from well-known hospitals in London and the provinces, and many of them have been operated upon by surgeons of the very highest reputation, yet recurrence has taken place quickly or slowly in a diffuse "cuirasse" or discrete nodular form. Granted that a certain number of these cases were, at the time of operation, not very advanced, it becomes evident that in some way or other the "radical" operation was not radical enough.

The observance of a number of cases which presented modular recurrences about the lower half of the scar led me to believe that in certain instances such recurrences might be due to the almost invariable practice of leaving intact the serratus magnus muscle, perhaps already infected by outlying extensions of growth.

Whilst removal of the pectoralis major is now an accepted routine, and removal of the pectoralis minor is a debatable point, scant attention has been paid to the serratus magnus, a muscle which from its anatomical relation to the breast demands the closest investigation. Both Sir Henry Morris ${ }^{1}$ and Handley ${ }^{2}$ advocate occasional removal of portions of this muscle, not, however, with quite that emphasis which on anatomical grounds would appear imperative. So far as I am aware, no microscopical evidence has been adduced as to the infection or non-infection of this muscle in certain cases of mammary cancer, and it was to gain evidence on this point that this research was carried out.

It is an anatomical fact that normally one.third of the female breast lies on the fascia over the serratus magnus. This corresponds, in the main, to the lower and outer third of the breast, and to the digitations of the muscle arising from the fifth and sixth ribs.

It is uncommon for the lower limit of the breast to reach below the level of the sixth costal arch. The four lowest digitations of the muscle at their origin are covered only by fascia and skin, and in the female the fifth and sixth digitations are covered also directly by the normally developed breast. Above this level the breast lies almost entirely upon the pectoralis major. It is not unusual for the breast to have more extensive margins than is apparent, and to have outlying isolated portions; but it is probable that, in the very large majority of cases, no ,portion of the organ lies directly upon the upper serrations of the muscle. The field of inquiry was thus limited to the lower serrations, especially those directly underlying the breast-namely, those arising from the fifth and sixth ribs. The muscle is naturally divisible into three portions:

1. A muscular bundle from the first and second costal arches, which is inserted into the ventral aspect of the upper angle of the scapula.
2. A bundle from the second, third, and fourth ribs, which is inserted into the whole of the ventral aspect of the vertebral border of the scapula between the upper and lower angles.

3. A bundle from the fifth, sixth, seventh, and eighth ribs is inserted into the ventral aspect of the inferior angle.

The innervation of the muscle follows also this muscular and anatomical division, the three bundles being supplied, subject to occasional variation, by the fifth, sixth, and seventh cervical nerves respectively. I have proved to my own entire : satisfaction that removal of the lowest bundle (from the fifth, sixth, seventh, and eighth ribs) does not impair the functional activity of the arm, nor the nervous supply of the upper two segments of the muscle, and it would appear that these two segments, with their extensive origin and insertion, are fully capable of carry. ing out the necessary scapular movements. Such removal is only a step further than the recognized routine of removal of the serratus fascia; it adds nothing to the difficulties of the operation, and takes but a minute or two to perform. The serrations can be detached from their origin, stripped back and divided close to their insertion. The lowest fasciculus of the pectoralis minor is a guide to the fifth rib.

In view of the proved fact that the pectoralis major is frequently infected by the "lymphatic permeation" of Handley long before any clinical ubservations of the attachment of the growth to the muscle beneath it can be demonstrated, it appeared reasonable to conclude that a similar infection might be shown to be present in another directly underlying muscle, and that, moreover, such an investigation might give some clue to the problem of recurrence. Though the results have been negative, it has seemed right to record the investigation as far as it goes, in the hope that the subject may be more fully pursued by others who also see a large number of mammary cancers.

The cases selected were all carefully picked from amongst some 150 mammary tumours successively admitted to the Cancer Hospital. These picked cases are only 8 in number, but in these 8 cases either the growth was lying directly over the serratus magnus, or the whole breast was involved, including that portion lying over this muscle. All the cases were submitted to operation, and in all the tumour was proved microscopically to be carcinoma. No inoperable case was investigated, as it was desired to exclude as far as possible any case in which the muscle might have become directly infiltrated by coarse invasion in contradistinction to lymphatic permeation; my object being to determine the condition of the muscle as regards infection while the growth was still operable.

At first cases were selected which showed no clinical evidence that the growth was tacked on to the fascia beneath it, but later, as no muscular infection could be demonstrated in these cases, cthers were investigated in which the growth had only a limited mobility over the serratus fascia.

My colleagues in their own cases were most kind in removing such portions of muscle as I required in tine manner I wished. The method adopted was, as far as possible, to peel off the pectoralis major, serratus fascia, and lowest segment of serratus muscle in a continuous sheet. The relative position of the tumour, fascia, and muscle was then reconstructed, a selected portion hardened in the natural position of the parts, and a number of sections in series cut and examined. At this stage Dr. Alex. Paine and Dr. Kettle, of the Cancer Research Department of the hospital, afforded me most valuable help, for which I wish to express my gratitude and indebtedness to them.

In not one of the cases could any trace of cancer cells be found in a numerous series of muscle sections, although processes of cells were visible extending in the fascia right up to the muscle margin; nor was there any evidence of the irritative round.celled infiltration surrounding a cancerous lymphatic, nor any sign of a degenerated cancer plug, obliterated by a perilymphatic fibrosis.

These results are surprising, but, I believe, are destined to be reversed in the future. If there are lymphatics in the pectoralis major which communicate at an angle with those in the pectoral fascia, the same arrangement is more than likely to hold good in the case of the serratus magnus, and it will be found in the future to be a routine necessity to remove the "exposed" portion of this muscle in the 